\title{
Modelling of Asymmetric Rotor and Cracked Shaft
}

\author{
Tatsuro Ishibashi ${ }^{1}$ Atsushi Yoshida ${ }^{2}$ Tadao Kawai ${ }^{2}$ \\ ${ }^{1}$ Meidensha Corporation, Japan, ishibashi-tat@mb. meidensha . co.jp \\ ${ }^{2}$ Department of Mechanical \& Physical Engineering, Osaka City University, Japan, \\ m17ta040@ka.osaka-cu.ac.jp, kawai@mech.eng.osaka-cu.ac.jp
}

\begin{abstract}
The object of this paper is to present asymmetric rotor and shaft models in rotating machinery systems. Using these models it is possible to analyze the electrical motors or generators which have different lateral stiffness or the moments of inertia in two orthogonal directions. The asymmetry causes unstable vibrations in some rotating speed ranges. A cracked shaft model is presented as the extension of the asymmetric shaft model. These models are implemented in our original rotating machinery library.
\end{abstract}

Keywords: Rotor Dynamics, Asymmetrical Rotor, Asymmetrical Shaft, Cracked Shaft

\section{Introduction}

Rotating machinery systems have been dominant in most of heavy equipment such as turbine, generator, motor and so forth. Rotating machinery systems are always the key component of these equipment and the dynamic properties of which can determine the performance of the whole system. Before manufacturing, the modelling and simulation of rotating machinery systems can help the engineers to design a better system. Rotating machinery systems may suffer from different kinds of faults. A proper modelling and simulation of rotating machinery system with common faults should help engineers understand the performance with faults well, so that faults can be discovered or diagnosed in the early stage. Approaches to dynamic analysis of rotor systems can be divided into two main branches. One is the widely used finite element method (FEM), and the other is the relatively more traditional one, i.e. the transfer matrix method (TMM). This method is relatively simple and straightforward in application. The rotating shaft are decomposed into the concentrated mass and flexible beam. The main advantage of TMM is low computational cost.

Modelica is an object-oriented, declarative, acausal, multi-domain modelling language for componentoriented modelling of complex systems. It is suitable for modelling and simulation of rotating machinery systems with faults, whose parameters are frequently changed. Causal modelling method appears to be inefficient because the code is difficult to modify and reuse. Modelling by Modelica decomposes rotating machinery systems into several basic components. Basic components are reusable, and their parameters can be simply modified. By using the Modelica_LinearSystems2 library, it is possible to do eigenfrequency analysis in the linearized system. These are the big advantage for modelling and simulating of the rotating machinery systems with several faults by TMM.

Several papers have been written relating modelling rotor dynamics in Modelica. Vibrations of gears in the plane have been implemented and refined (Dahl et al, 2017; Kosenko and Gusev, 2012; van der Linden, 2012). However those are focusing on only a gear. Previously $3 \mathrm{DOF}$ (degree of freedom) rotor dynamics library with multi-faults is reported (Ming et al, 2013). It only handle Jeffcott rotor systems neglecting the gyroscopic effect, which is very important for rotor dynamics.

We have created the rotating machinery library which has 5 DOF rotor dynamics model components (Ishibashi et al, 2017). The library has common faults of rotating machinery systems including static and dynamic unbalance, shaft bending, and faulty bearing. In this paper, we implemented asymmetrical rotor and shaft models. The actual rotating machinery systems have asymmetries such as two pole generators and propellers etc. The cracked shaft model is also implemented as the extension of the asymmetric shaft model.

\section{Asymmetrical systems}

The actual rotating machinery such as propeller rotors and two-pole generator rotors have the asymmetry. The former are systems with a directional difference in the rotor inertia (Figure 1 (a)). The latter are systems with a directional difference in the shaft stiffness (Figure 1 (b)). As the shaft with these directional differences rotates, terms with time-varying coefficients appear in the governing equations. The most characteristic property of asymmetrical system is the appearance of unstable vibrations in some rotating speed ranges (Ishida and Yamamoto, 2012; Matsushita et al, 2017). The asymmetrical rotor and shaft models are implemented in our rotating machinery library. 


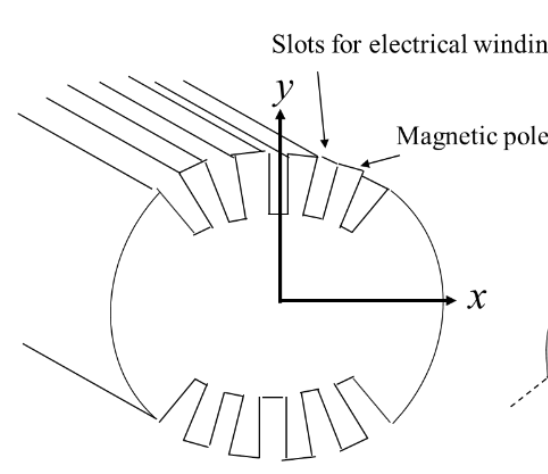

(a) A generator rotor

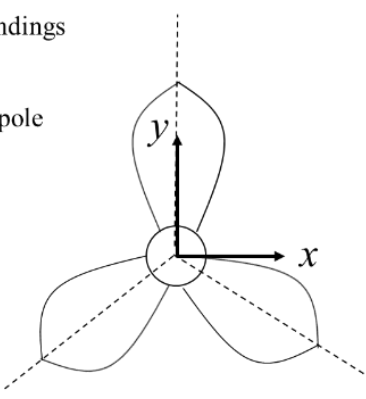

(b) A three-bladed propeller

Figure 1. Asymmetries of the rotor and the shaft.

\subsection{Asymmetrical rotor}

The asymmetrical rotor is considered as a single point with a rigid disc or a long rigid shaft. The directional difference in the moment of inertia is expressed as $I_{1}=I+\Delta I, I_{2}=I-\Delta I$. The equations of asymmetrical rotor motion are following.

$$
\begin{aligned}
& I \ddot{i_{x}}+I_{p} \dot{\theta} \dot{i_{y}}-\Delta I \frac{d}{d t}\left(\dot{i_{x}} \cos 2 \omega t+\dot{i_{y}} \sin 2 \omega t\right) \\
& +\tau \dot{\theta}^{2}\left\{\left(I-I_{p}\right) \cos \left(\omega t+\tau_{0}\right)-\Delta I \cos \left(\omega t-\tau_{0}\right)\right\} \\
& =M_{x} \\
& I \ddot{i_{y}}-I_{p} \dot{\theta} \dot{i_{x}}-\Delta I \frac{d}{d t}\left(\dot{i_{x}} \sin 2 \omega t+\dot{i_{y}} \cos 2 \omega t\right) \\
& +\tau \dot{\theta}^{2}\left\{\left(I-I_{p}\right) \sin \left(\omega t+\tau_{0}\right)-\Delta I \sin \left(\omega t-\tau_{0}\right)\right\} \\
& =M_{y}
\end{aligned}
$$

Here,

$i_{x}, i_{y}$ : Deflection angle of each direction,

$M_{x}, M_{y}$ : Moment of each direction,

$\theta$ : Rotational angle,

$\tau$ : Slope of dynamic unbalance,

$\tau_{0}$ : Initial phase of dynamic unbalance,

$\Delta I$ : Difference of moment of inertia,

$I$ : Average moment of inertia,

$I_{1}$ : Moment of inertia about $y$ axis,

$I_{2}:$ Moment of inertia about $x$ axis,

$I_{p}:$ Polar moment of inertia.

\subsection{Asymmetrical shaft}

According to the coordinate system $\mathrm{O}-\xi \eta$ rotating with the shaft shown in Figure 2, the asymmetrical elastic shafts which have a directional difference in the shaft stiffness, holds the following equation.

$$
\begin{aligned}
E\left(\begin{array}{c}
I_{\xi} i_{b \xi} \\
I_{\eta} i_{b \eta}
\end{array}\right) & =\frac{L^{2}}{2}\left(\begin{array}{c}
F_{a \xi} \\
F_{a \eta}
\end{array}\right)+L\left(\begin{array}{c}
M_{a \xi} \\
M_{a \eta}
\end{array}\right)+E\left(\begin{array}{c}
I_{\xi} i_{a \xi} \\
I_{\eta} i_{a \eta}
\end{array}\right) \\
& +\left(\begin{array}{cc}
\cos \theta & -\sin \theta \\
\sin \theta & \cos \theta
\end{array}\right) \times\left(\begin{array}{c}
0 \\
-\frac{m g L^{2}}{6}
\end{array}\right)
\end{aligned}
$$

$$
\begin{gathered}
E\left(\begin{array}{c}
I_{\xi} u_{b \xi} \\
I_{\eta} u_{b \eta}
\end{array}\right)=\frac{L^{2}}{6}\left(\begin{array}{c}
F_{a \xi} \\
F_{a \eta}
\end{array}\right)+\frac{L}{2}\left(\begin{array}{c}
M_{a \xi} \\
M_{a \eta}
\end{array}\right)+E L\left(\begin{array}{c}
I_{\xi} i_{a \xi} \\
I_{\eta} i_{a \eta}
\end{array}\right) \\
+E\left(\begin{array}{c}
I_{\xi} u_{a \xi} \\
I_{\eta} u_{a \eta}
\end{array}\right)+\left(\begin{array}{cc}
\cos \theta & -\sin \theta \\
\sin \theta & \cos \theta
\end{array}\right) \times\left(\begin{array}{c}
0 \\
-\frac{m g L^{3}}{24}
\end{array}\right)
\end{gathered}
$$

The second moments of area changes twice per revolution at the stationary coordinate system as expressed by Equation 5 .

$$
\left(\begin{array}{l}
I_{x} \\
I_{y}
\end{array}\right)=\frac{I_{\xi}+I_{\eta}}{2}\left(\begin{array}{l}
1 \\
1
\end{array}\right)+\frac{I_{\xi}-I_{\eta}}{2} \cos 2 \omega t\left(\begin{array}{c}
-1 \\
1
\end{array}\right)
$$

Here,

$m$ : Shaft mass,

$L:$ Shaft length,

$E$ : Young's modulus,

$I_{x}, I_{y}$ : Second moments of area in the stationary coordinate system,

$I_{\xi}, I_{\eta}$ : Second moments of area in the rotating coordinate system,

$g:$ Constant of gravitation,

$\omega$ : Rotating speed.

The indices $a$ and $b$ mean left and right flange respectively.

This time-varying coefficients cause an unstable range in machine operating frequency.

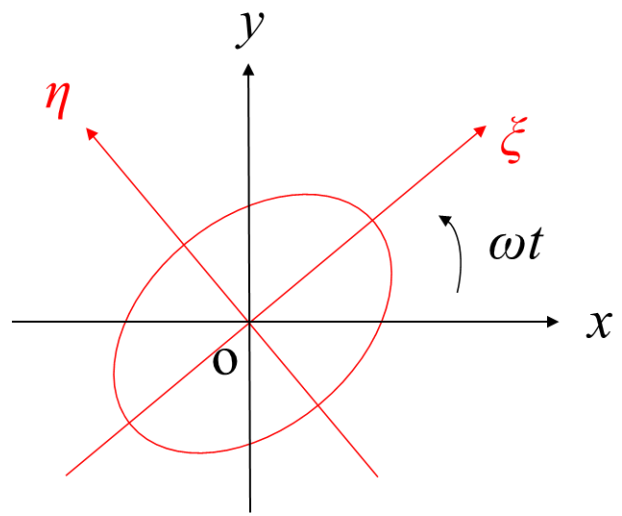

Figure 2. The stationary (black) and rotating coordinate system (red).

\section{Cracked shaft}

One of the most important causes of accidents in rotating machinery is the crack caused by fatigue. In horizontal shaft system, since the weight of the rotors and shafts bend shafts, expanding force acts on the underside of the shaft and compressing force acts on the upper side. As the result, when the shaft rotates, periodic stress is generated and cracks sometimes occur. In the cracked shaft, the stiffness differs depending on the direction in which the shaft bends. In the crack opening direction, the shaft stiffness is small, and on the other hand, in the closing direction, the stiffness becomes large (Figure 3 (a)). The restoring force of the shaft has a nonlinear spring characteristic in a fragment linear fashion along the $\eta$ direction at the rotating coordinate 
system $\mathrm{O}-\xi \eta$ rotating with the shaft (Figure $3(\mathrm{~b}))$. Along the $\xi$ direction, the restoring force has a linear spring (Gasch, 1976; Henry and Okah-Avae, 1976). The cracked shaft model is expressed as the extension of the asymmetrical shaft model. In Equation 3 and 4, the second moments of area at the rotating coordinate system changes as follows.

$$
I_{\eta}= \begin{cases}I_{\eta}-\delta, & i_{b \eta}-i_{a \eta} \geq 0 \\ I_{\eta}+\delta, & i_{b \eta}-i_{a \eta}<0\end{cases}
$$

Here,

$\delta$ : Crack strength parameter. (a)

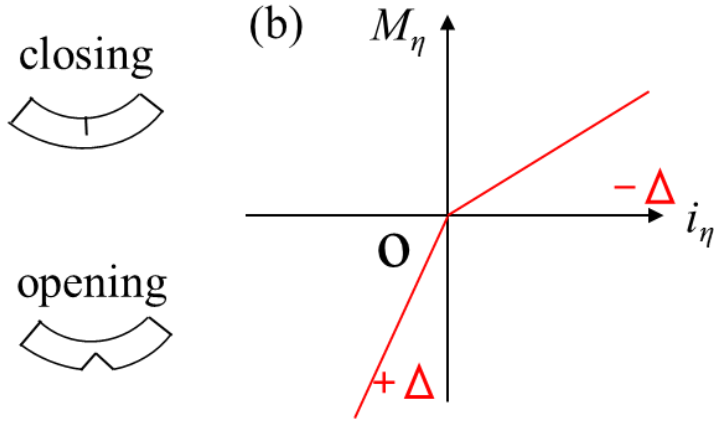

Figure 3. The cracked shaft model. (a) Conceptual diagram (b) A nonlinear spring characteristic in a fragment linear fashion.

\section{Example Models}

The above models are using our original rotating machinery library. Dymola is used for the simulations. The asymmetrical rotor model is implemented by modifying rotor. The asymmetrical and cracked shaft models are implemented by modifying the shaft. Figure 4 shows a simple Jeffcott rotor model using our rotating machinery library. Here, we simulate the asymmetrical rotor and shaft and the cracked shaft in the Jeffcott rotor system.

\subsection{Symmetrical system}

Before the transient simulation, we check the symmetrical model by eigenfrequencies analysis using the Modelica_LinearSystems2 library (Bauer et al, 2009; Otter, 2006) and creating the function. Figure 5 shows the Campbell diagram. Red curve shows the

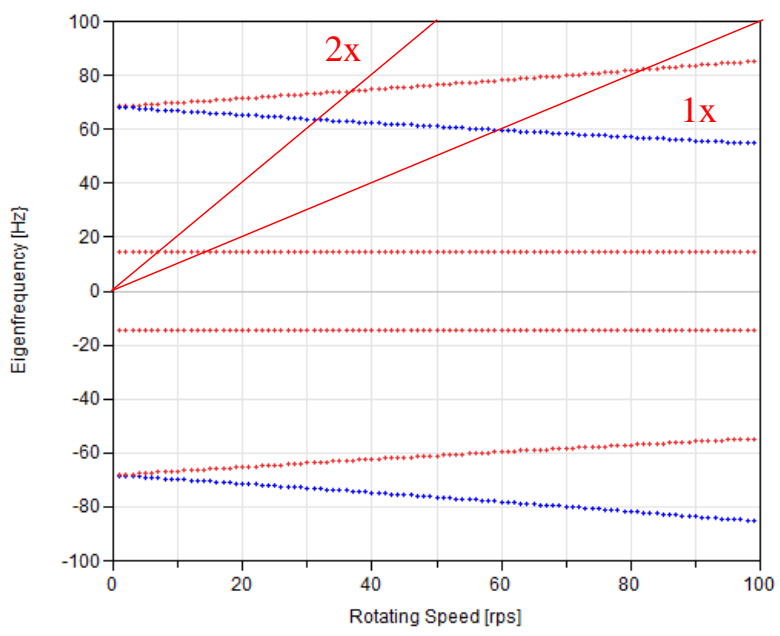

Figure 5. The Campbell diagram of the symmetrical system. Red shows the counterclockwise rotation whirl mode. Blue shows the clockwise rotation whirl mode.

counterclockwise rotation whirl mode and blue curve shows the clockwise rotation whirl mode by eigenvector analysis. The eigenfrequency curves starting from around $70 \mathrm{~Hz}$ split due to the gyroscopic effects. The intersection between the synchronous excitation line and the eigenfrequencies in Campbell diagrams are referred as critical speeds. Also, the intersection between twice the synchronous excitation line and the eigenfrequencies are referred as secondary critical speeds. This system has two critical speeds around $14 \mathrm{~Hz}$ and $83 \mathrm{~Hz}$. Since it is easy to understand the simulation results, in this case, time derivatives terms in the equations of journals motion are ignored.

To check this system, we simulate the ramp response of this system. Rotor with a diameter of $80 \mathrm{~mm}$ has both the static and dynamic unbalance. Shaft3 with a diameter of $8 \mathrm{~mm}$ also has the shaft bending. Figure 6 and Figure 7 show the deflection in the horizontal direction and the deflection angle vibration of Rotor against the rotating speed respectively. The rotating speed is raised from 0 rps to $100 \mathrm{rps}$ at the rate of 10 $\mathrm{rps} / \mathrm{s}$ by replacing the constant input with the ramp input in Figure 4. The deflection has the peak at the a little bit higher speed of the $15 \mathrm{rps}$ and the deflection angle has the peak around $83 \mathrm{rps}$.

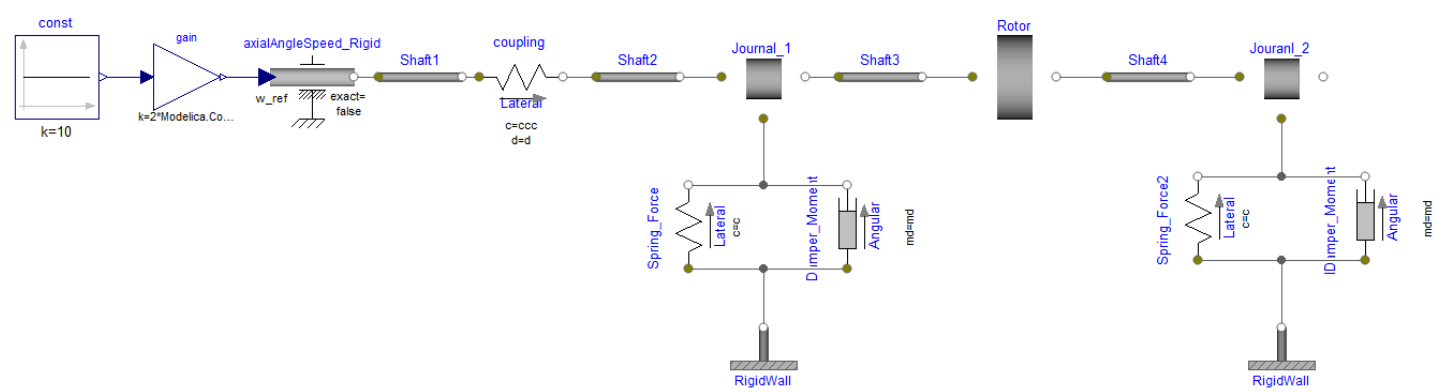

Figure 4. Modelica model of a Jeffcott rotor system. 


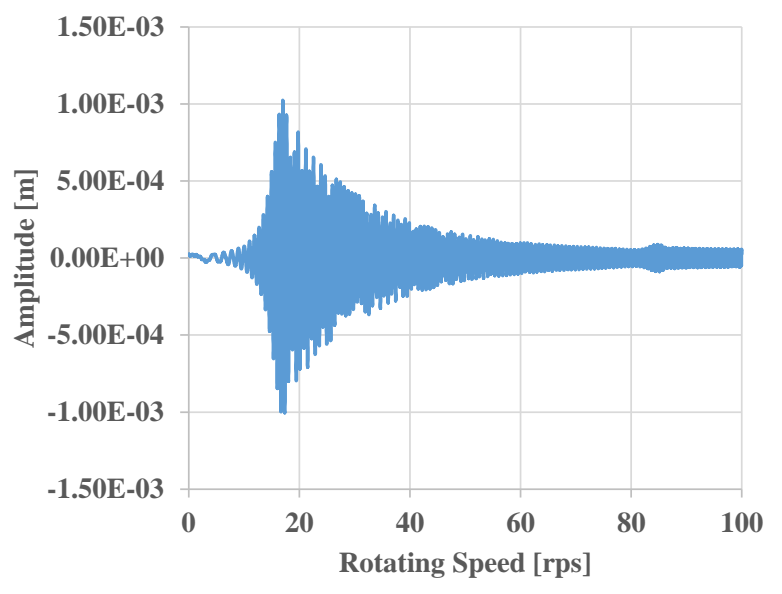

Figure 6. Ramp response of Rotor deflection.

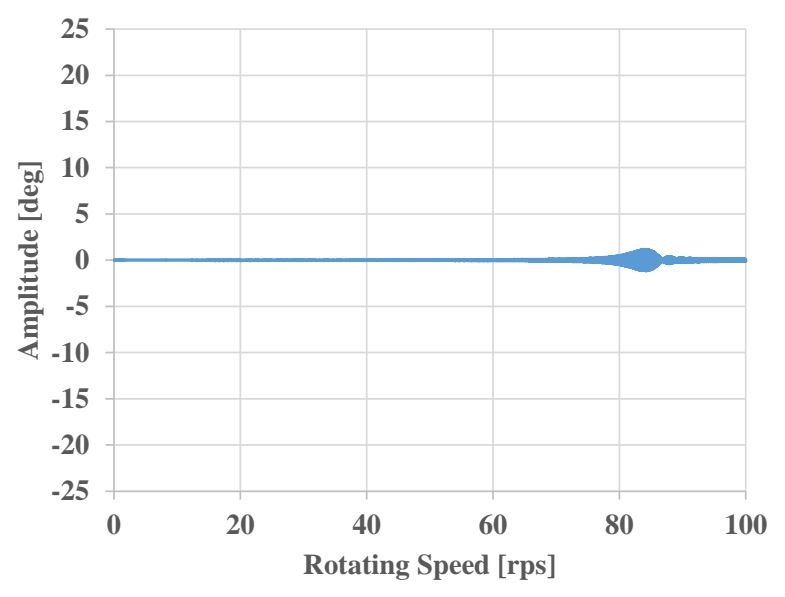

Figure 7. Ramp response of Rotor deflection angle.

\subsection{Asymmetrical system}

\subsubsection{Asymmetrical rotor}

We simulate the model replacing Rotor with the asymmetrical rotor in Figure 4. The asymmetrical rotor is expressed in Equation 1 and 2. The asymmetrical rotor is assumed as the elliptic cylinder with the long axis of $45 \mathrm{~mm}$ and the short axis of $35 \mathrm{~mm}$ which has the directional difference in the moment of inertias $\Delta I=$ $6.4 \mathrm{e}^{-4} \mathrm{kgm}^{2}$.

Figure 8 and Figure 9 show the ramp response. The deflection angle peak amplitude is larger than that of the symmetrical system, although the peak positions in both the deflection and deflection angle are the totally same positions as the symmetrical system. This implies that the rotating speed around $83 \mathrm{rps}$ is unstable. To examine the unstable region, we simulate the model at several constant rotating speeds around 83 rps.

Figure 10 shows the transient simulation results of rotor deflection angles at the different constant rotating speeds respectively. At the rotating speed of 80 and 86 rps, Rotor deflection angle converges. From 81 to 85 rps, Rotor deflection angle vibration diverges. At 83 rps, Rotor deflection angle diverges rapidly. These clearly indicate that this system is unstable in this region. Unstable regions are demonstrated in the asymmetrical rotor system, whereas in the symmetrical system they are not observed. Due to the directional difference in the moment of inertias, the unstable region exists only in this region.

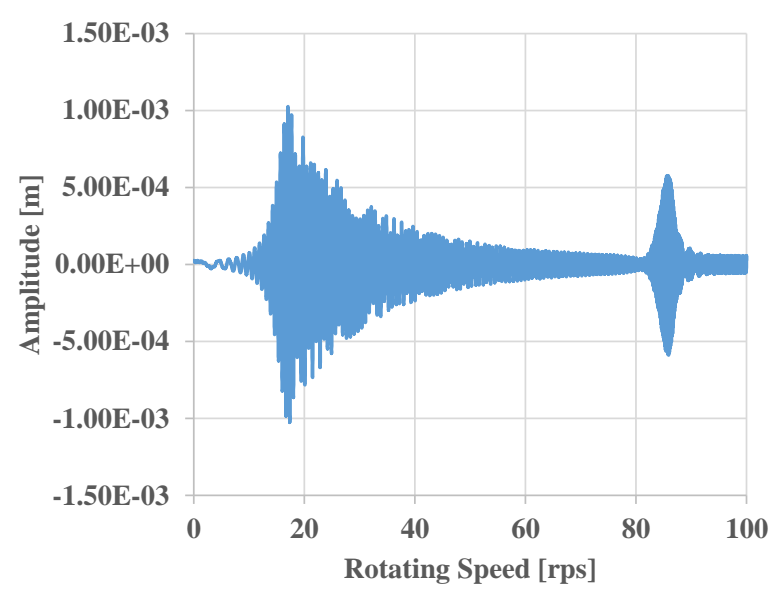

Figure 8. Ramp response of Rotor deflection with the asymmetrical rotor.

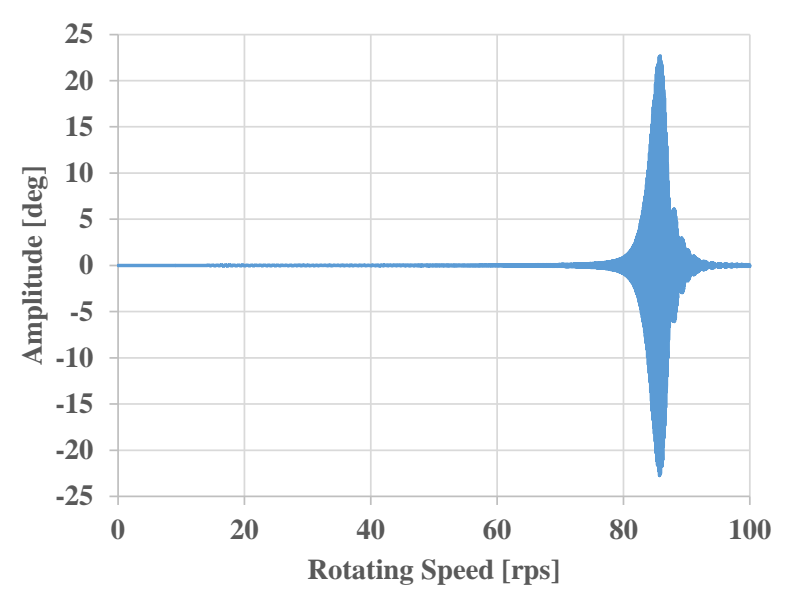

Figure 9. Ramp response of Rotor deflection angle with the asymmetrical rotor.

\subsubsection{Asymmetrical shaft}

We simulate the model replacing Shaft3 with the asymmetrical shaft in Figure 4. The asymmetrical shaft model is expressed in Equation 3, 4 and 5. The asymmetrical shaft is assumed as the elliptic cylinder with the long axis of $4.5 \mathrm{~mm}$ and the short axis of $3.5 \mathrm{~mm}$ which has the directional differences in second moments of area. 

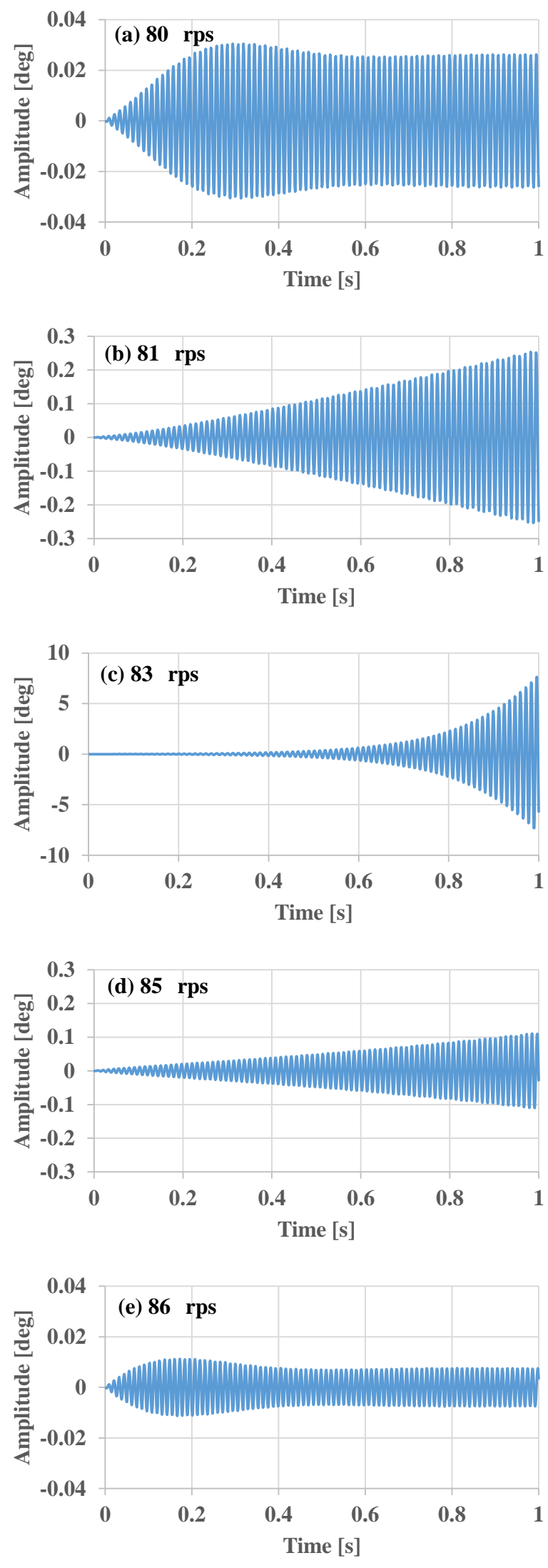

Figure 10. Unstable regions of the asymmetrical rotor system. Transient results of Rotor deflection angle with the asymmetrical rotor at different constant speeds.
Figure 11 and Figure 12 show the ramp response. The behavior is the same as the asymmetrical rotor system. Since the equations of motion of both the deflection and deflection angle have the directional stiffness differences in the asymmetrical shaft system, the asymmetrical shaft system has much more unstable regions such as the region around $14 \mathrm{rps}$.

To examine the unstable region, we simulate the model at several constant rotating speeds around $14 \mathrm{rps}$. Figure 13 shows the transient simulation results of Rotor deflections in the horizontal direction at the different constant rotating speeds respectively. At 14.1 and 14.7 rps, Rotor deflection converges. From 14.2 to $14.6 \mathrm{rps}$, Rotor deflection diverges. At $14.6 \mathrm{rps}$, Rotor deflection diverges rapidly. These clearly indicate that the system is unstable in this region. Similarly the system is unstable at the region around $83 \mathrm{rps}$. Also, the other several unstable regions corresponding to the minute peaks at 45 and 62 rps exist in simulation.

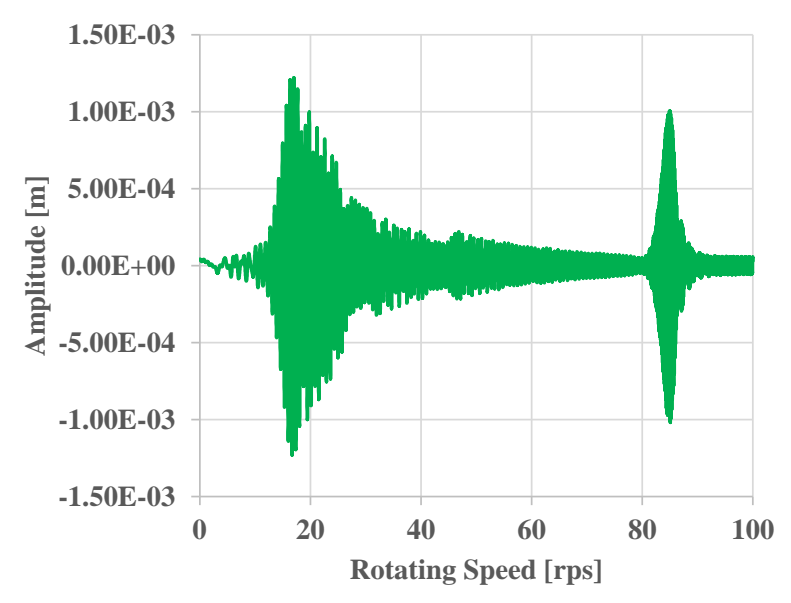

Figure 11. Ramp response of Rotor deflection with the asymmetrical shaft.

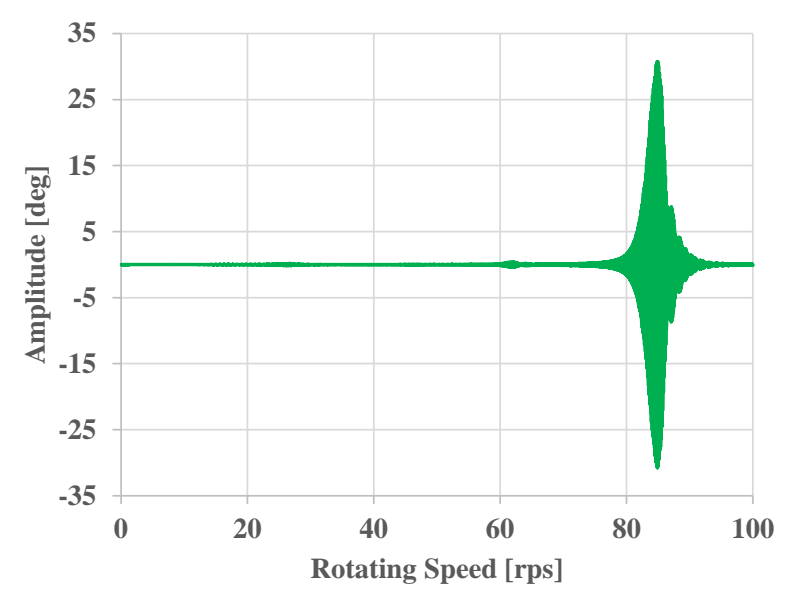

Figure 12. Ramp response of Rotor deflection angle with the asymmetrical shaft. 

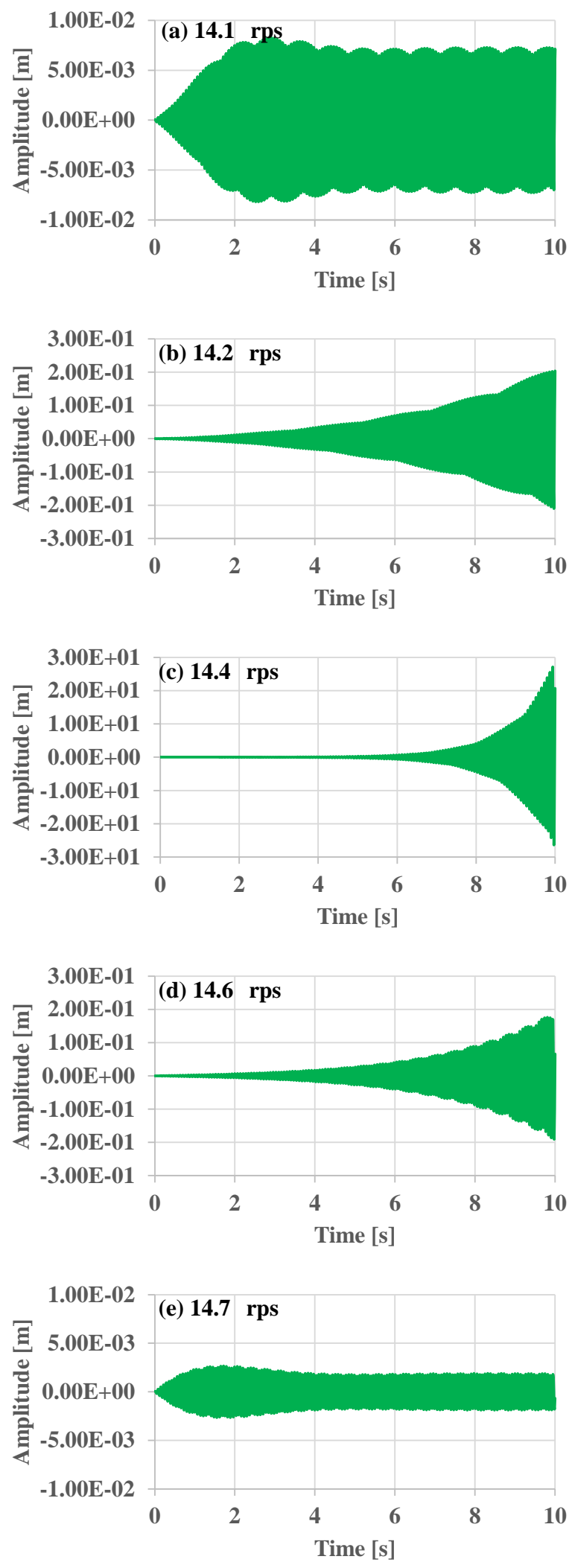

Figure 13. Unstable regions of the asymmetrical shaft system. Transient results of Rotor deflection with the asymmetrical shaft at different constant speeds.

\section{Cracked shaft}

We simulate the model replacing Shaft3 with the cracked shaft in Figure 4. The cracked shaft model is expressed as the extension of the asymmetrical shaft by adding Equation 6. Here, we examine the nonlinear vibration of the cracked shaft. The cracked shaft shape is symmetrical. It has the crack strength parameter $\delta$ with half the second moment of the area of the symmetrical shaft.

The asymmetrical shaft and the symmetrical system are simulated to check the differences. Figure 14 shows the transient simulation results of the rotor deflection angle at the constant rotating speed of $5 \mathrm{rps}$.

We analyze frequency characteristics of results by FFT signal processing with a rectangular window in the range of 4 to $6 \mathrm{~s}$. The range is chosen to remove the initial transient effect of this system. Figure 15 shows the results of FFT analysis. The symmetrical system has the only component synchronized with the rotating speed. The asymmetrical shaft has the other components. The frequency of $14 \mathrm{~Hz}$ is the eigenfrequency of the
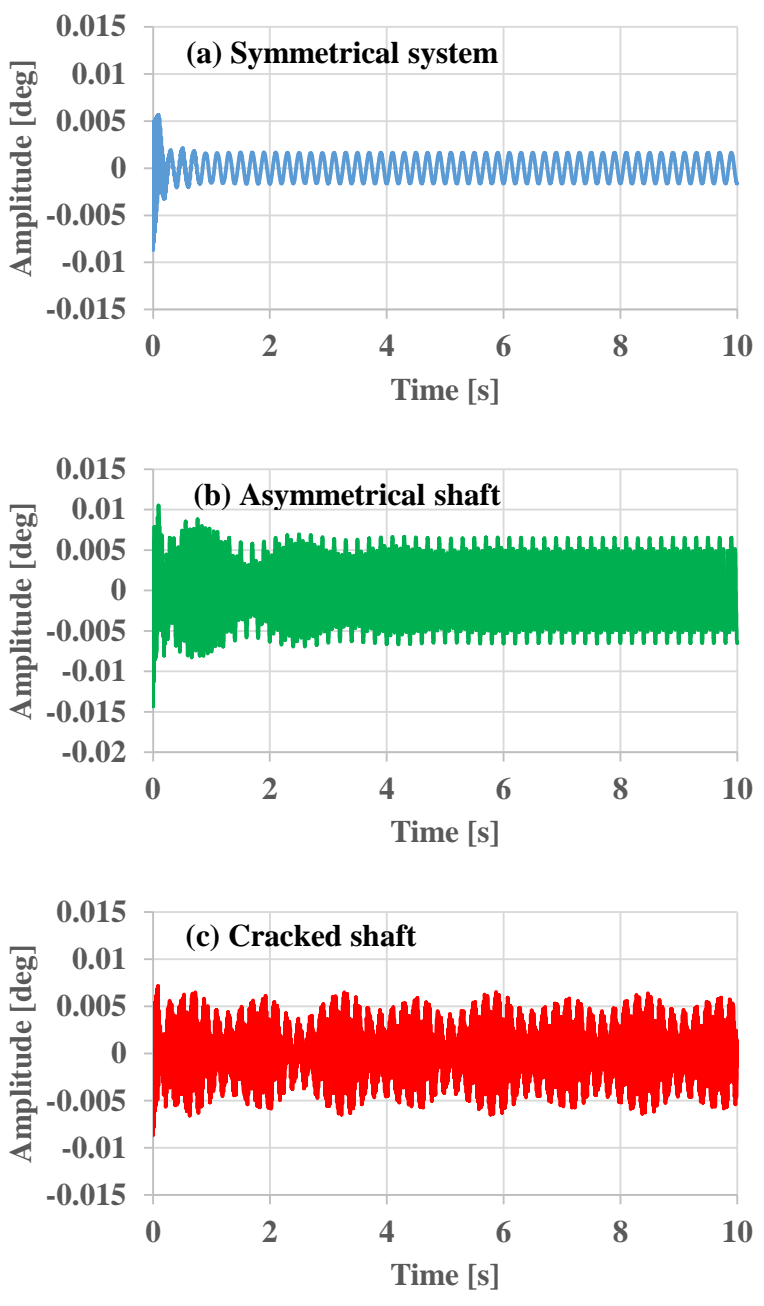

Figure 14. Comparison of transient simulation results at constant speed 5 rps. (a) Symmetrical system. (b) Asymmetrical shaft. (c) Cracked shaft. 


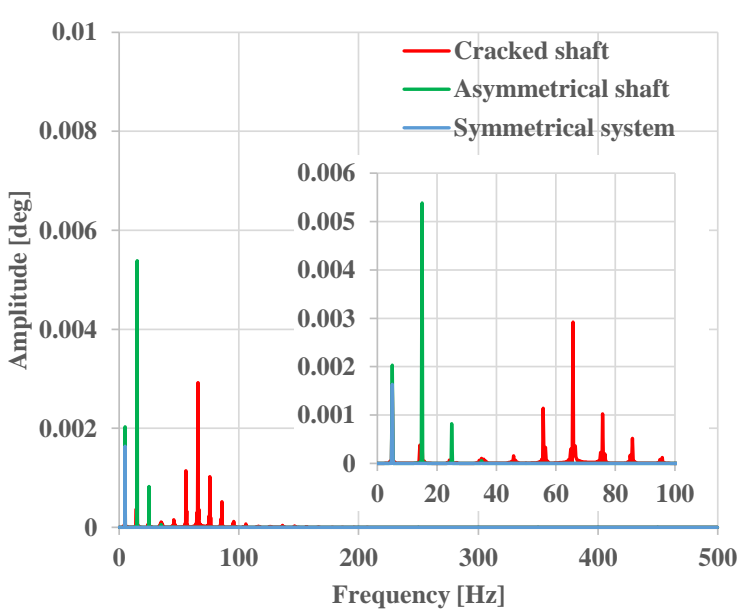

Figure 15. Comparison of FFT analysis. Blue line: Symmetrical system. Green line: Asymmetrical shaft. Red line: Cracked shaft. The inset shows the enlarged view.

rotor horizontal vibration mode in this system. The cracked shaft has the same components as the asymmetrical shaft and the other components due to the nonlinear spring characteristic in a fragment linear fashion.

\section{Conclusion}

In this paper, models are presented to simulate the asymmetrical rotor and shaft and the cracked shaft. Using our rotating machinery library, it is possible to model an actual rotating machinery such as propeller rotors and two-pole generator rotors with asymmetries. As the extension of the asymmetrical shaft model, the cracked shaft model is implemented. Examples of simple rotor systems are demonstrated. The presented model shows the abilities to design and diagnose rotating machinery systems.

\section{References}

Markus Dahl, Håkan Wettergren and Henrik Tidefelt. Modelica Spur Gears with Hertzian Contact Forces. Proceedings of the 12th International Modelica Conference, 2017. doi:10.3384/ecp17132755.

Robert Gasch. Dynamics Behavior of a Simple Rotor with a Cross-Sectional Crack. Proceedings of the International Conference on Vibrations in Rotating Machinery, I. Mech. E. 123-128, 1976.

T. A. Henry and Okah-Avae. B. E. Vibrations in Cracked Shaft. Proceedings of the International Conference on Vibrations in Rotating Machinery, I. Mech. E. 15-17, 1976.

Tatsuro Ishibashi, Han Bing and Tadao Kawai. Rotating Machinery Library for Diagnosis. Proceedings of the 12th International Modelica Conference, 2017. doi:10.3384/ecp17132381.

Yukio Ishida and Toshio Yamamoto. Linear and Nonlinear Rotordynamics: A Modern Treatment with Applications, 2nd Edition. Wiley-VCH, 2012.
Ivan Kosenko and Ilya Gusev. Revised and improved implementation of the spur involute gear dynamical model. Proceedings of the 9th International Modelica Conference, 2012. doi:10.3384/ecp12076311.

Osami Matsushita, Masato Tanaka, Hiroshi Kanki, Masao Kobayashi and Patrick Keogh. Vibrations of Rotating Machinery. Springer Japan, 2017. doi: 10.1007/978-4-43155456-1.

Li Ming, Wang Yu, Li Fucai, Li Hongguang, and Meng Guang. Modelica-based Object-orient Modeling of Rotor System with Multi-Faults. Chinese Journal of Mechanical Engineering, Vol. 26, No. 6, 2013.

F.L.J. van der Linden. Modelling of elastic gearboxes using a generalized gear contact model. Proceedings of the 9th International Modelica Conference, 2012. doi:10.3384/ecp12076303. 